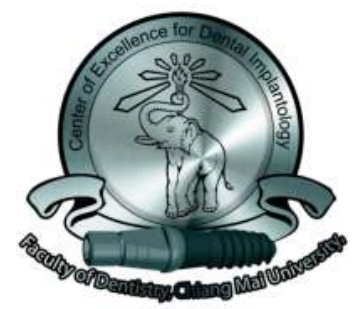

\title{
Comparative study of immediate loading on short dental implants and conventional dental implants in the posterior mandible : A randomized clinical trial four months post-loading.
}

\author{
Kritsada Weerapong, Pathawee Khongkhunthian*
}

\section{Center of Excellence for Dental Implantology, Faculty of Dentistry, Chiang Mai University}

Immediate dental implant placement and loading treatment protocol has been investigated with the promising clinical results. However, the use of short implant for immediate loading after surgical implant placement has not been investigated. With the trending CAD-Cam technology, the implant placement together with immediate restoration is possible to perform in dental clinic.

The purpose of the study was to compare the clinical outcomes and survival rates of immediately-loaded short implants and conventional length dental implants (Pw Plus dental implant system, Thailand) in replacing mandibular molar teeth.

\section{Patients and methods}

The clinical study was approved by the Human Experimentation Committee. Approval for the study was sought from the Human Experimentation Committee (TCTR20170802003) and was conducted at the Center of Excellence for Dental Implantology, Faculty of Dentistry, Chiang Mai University

Fifty implants in 46 patients were included in the study. Twenty five short dental implants $(6 \mathrm{~mm}$.) and 25 conventional dental implants $(10 \mathrm{~mm}$.) were randomly placed as single tooth replacements in the edentulous molar areas of the mandibles. Provisional, CAD-CAM, ceramic block (Shofu HC) crowns were cemented to the abutments of the implants, and immediately loaded. The clinical parameters, such as insertion torque (IT), resonance frequency analysis (RFA), marginal bone level changes, and complications, were recorded and statistically analyzed.
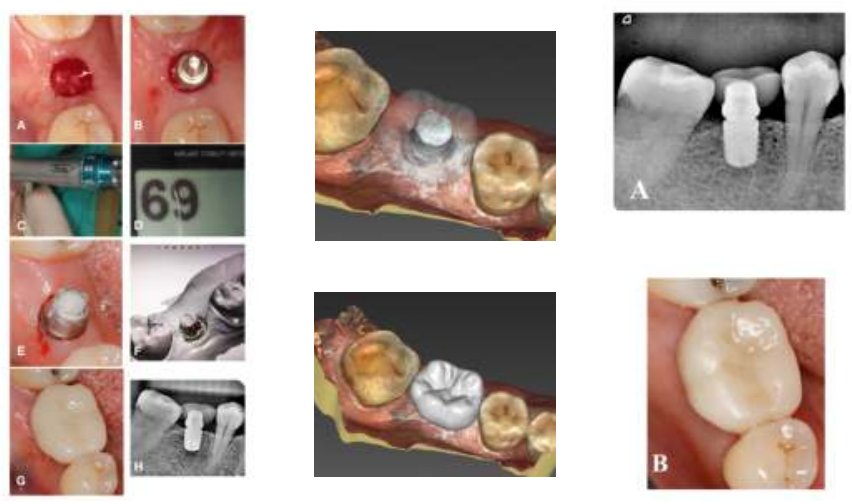

\section{Results}

Two short implants and two conventional implants were excluded from the study due to insufficient insertion torque (less than 35 $\mathrm{Ncm}$ ). Two short implants disintegrated, and one conventional implant failed. The cumulative survival rate of short implants was $91.30 \%$, compared to $95.65 \%$ for conventional implants. However, there was no significant difference between the two implant types $(\mathrm{P}$ (Fisher's exact $)=1.00)$. Only minor complications were found (three provisional crown fractures with short implants and two with conventional implants). The differences in IT among the two implant types were not significant $(P=.264)$. There was no significant difference in ISQ value for short or conventional implants between baseline (short: $73.86 \pm 2.38 \mathrm{~mm}$, conventional: $75.05 \pm 3.26 \mathrm{~mm}, \mathrm{P}=.088$ ) and four months after loading (short: $72.37 \pm 1.35 \mathrm{~mm}$, conventional: $72.89 \pm 1.87 \mathrm{~mm}, \mathrm{P}=.165)$. The mean change in marginal bone level four months post-loading was $0.24 \pm 0.29 \mathrm{~mm}$ for short implants and $0.26 \pm 0.29 \mathrm{~mm}$ for conventional implants; there was no statistical difference between the two implant types.

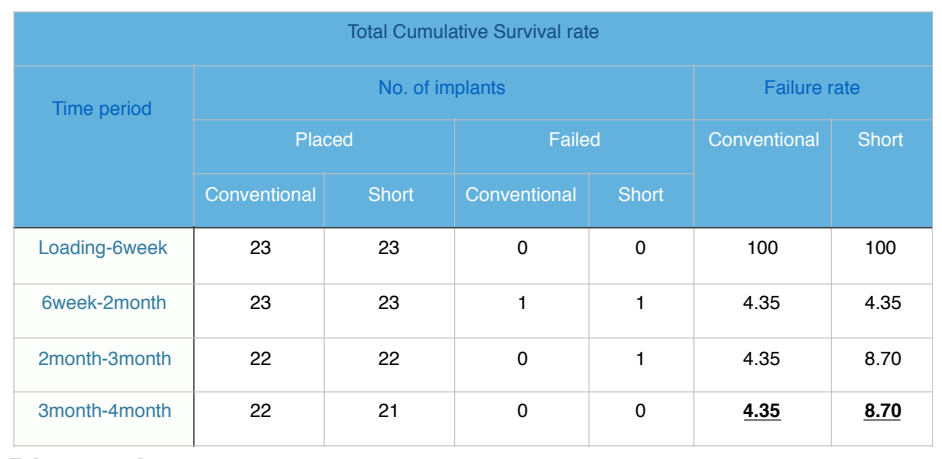

\section{Discussion}

Numerous studies have reported high success rate of immediate loading with standard length implant to affect single edentulous gap [1-3]. However, there are limited studies evaluating immediate loading of short implant. In 2008, Canizzaro et al. have reported the 4-year survival rate of immediate versus early loading of $6.5 \mathrm{~mm}$ short implant. The survival rate was $96.7 \%$ for both groups [4]. The meta-analysis of Srinivasan et al, 2013 reported that 6-mm-short implants demonstrated favorable survival rates and most of the failure occurred in first 4 month [5]. Therefore the 4 months follow-up period of our study could be indicated the success osteointegration of immediate loading implant placement. 